\title{
Distributed Particle Simulation Method on Adaptive Collaborative System*
}

\author{
Yudong Sun, Zhengyu Liang, and Cho-Li Wang \\ Department of Computer Science and Information Systems \\ The University of Hong Kong, Pokfulam Road, Hong Kong \\ \{ydsun, zyliang, clwang\}@ csis.hku.hk
}

\begin{abstract}
This paper presents a distributed $N$-body method based on an adaptive collaborative system model. The collaborative system is formed by the distributed objects in a cluster. The system can be reconfigured during the computation to fully utilize the computing power of the cluster. The method is implemented in Java and RMI to support distributed computing on heterogeneous platforms. A distributed tree structure is designed for communication-efficient computation of the method. The performance test shows satisfactory speedup and portability of the $\mathrm{N}$-body method on both of homogeneous and heterogeneous clusters. The collaborative system model is applicable to various applications and it is expandable to wide-area environment.
\end{abstract}

Keywords: $N$-body; Distributed object; Collaborative system; Java

\section{Introduction}

$N$-body problems study the evolution of a physical system with numerous bodies (particles) under the cumulative force influence on every body from all other bodies. The force influence causes continuous body movement. A lot of systems exhibit this behavior in astrophysics, plasma physics, molecular dynamics, fluid dynamics, radiosity calculations in computer graphics, and etc. [11]. The common feature of these systems is the large range of precision in the information requirements of the bodies in the physical domain. A body requires gradually rough information in less frequency from parts of the domain that are farther away. The body distribution in physical domain, therefore the domain parts and the influences from those parts are continuously changing during the evolution of the system. It needs to iterate the computation of the force influences on each body. Thus $\mathrm{N}$-body problems are computeintensive problems.

The methods for solving $N$-body problems $[1,5,6,7]$ are usually hierarchical methods in which a tree structure is applied to represent the body distribution in a physical domain. The tree is constructed based on domain decomposition. The force influences on the bodies are computed by traversing the tree. Barnes-Hut method $[1,11]$ is a typical hierarchical $N$-body algorithm. In the method a physical domain is recursively divided into sub-spaces until there is only one body (or none) in each sub-space. The domain decomposition is in accordance with the body distribution in the space. Fig. 1(a) gives an example on domain decomposition in $2 \mathrm{D}$ space. Then, a quadtree for $2 \mathrm{D}$ space is constructed based on the domain decomposition as Fig. 1(b) shows. For 3D space, an octree will be built. After the force on each body from all other bodies is computed by traversing the quadtree, the bodies move to their new positions under the force impact. That is one simulation step. The tree should be reconstructed in each

\footnotetext{
* This research was supported by Hong Kong Research Grants Council (RGC) grant 10201696 and The University of Hong Kong CRCG grant 10200544.
} 
step to reflect the updated body distribution. The particle simulation proceeds by iterating on tree construction and force calculation.

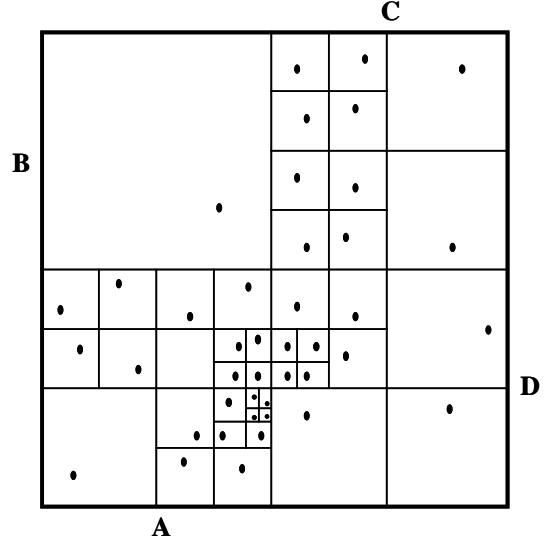

(a) Body distribution and domain decomposition

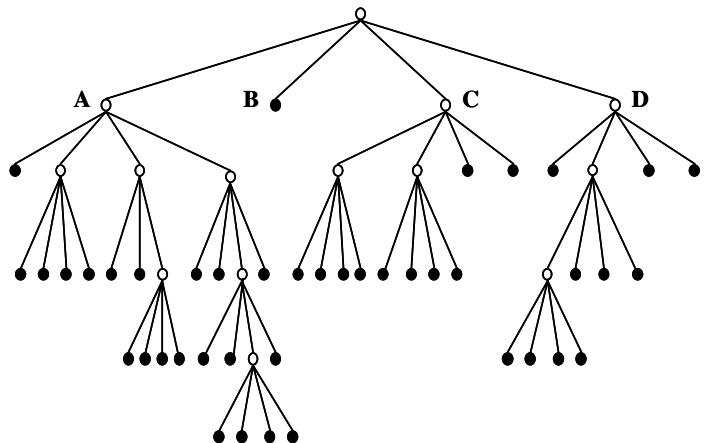

(b) Quad Barnes-Hut tree

Fig. 1 Barnes-Hut tree in 2D domain

Parallel $N$-body methods can be derived from the sequential Barnes-Hut method [4,10,11]. For example, Singh presented a parallel $N$-body method in shared-address-space model [11,12]. Multiple processes cooperatively build a global Barnes-Hut tree in shared memory segment. Then these processes carry out the force computation by concurrently traversing on the global tree. The sharedmemory model is only applicable in shared-memory systems like SMP machines in which data sharing can be realized by means of shared memory access. In distributed-memory systems, however, message passing is the general communication approach [4]. If the global tree approach is still used for solving $N$-body problems in distributed system, each process has to duplicate the global tree for its local access. The propagation of the global tree inevitably incur heavy communication. One solution to reduce the communication overhead is decomposing the global tree structure into subtrees. Each processor takes one of the subtrees. In this case, there is no need to build the global tree. With the proper sub-domain decomposition and data transferring schemes, inter-process communication can be cut down in the subtree scheme.

To solve $N$-body problem in distributed system, we have designed a distributed object-oriented (DOO) $\mathrm{N}$-body method based on collaborative system model. A collaborative system is formed by a group of distributed objects on the hosts in cluster. One of the objects works as the compute coordinator. It initiates the computing procedure by invoking the objects on remote hosts and dispatching computing tasks to these objects. It also collects the results of the computation afterwards. Other objects are called compute engine. Those objects accept and process the computing tasks. The $N$ body method in this paper is an improvement to the static method discussed in [13]. The method here is adaptive to the states of the underlying hosts. It allows the collaborative system to be reconfigurable. The computing tasks can be migrated from an overloaded host to a lightly-loaded one to enhance the computing efficiency. A distributed tree structure is constructed to support the communication-efficient computing of the $N$-body method in distributed system.

With the target to run on heterogeneous platforms, the $N$-body method is implemented in Java and RMI (Remote Method Invocation) [3]. Java is a platform-independent language that enables an application to directly run on various platforms. The object-oriented feature of Java strongly provides the foundation to the implementation of DOO method. RMI is a component of Java API. It is a Javabased interface to support distributed object-oriented computing. It supplies inter-object communication facilities that allows an object on one host to make method calls to remote objects on other hosts. RMI has a registry for remote object reference. Distributed objects can register themselves, locate each other and invoke the methods on remote objects through the registry [3]. These mechanisms 
in RMI make the collaborative system to be reconfigurable as well. When there are changes occurring in system resources (e.g., the available hosts in a cluster or their workloads are altered), the compute coordinator can select new hosts to join the computation and discard the stale ones. The objects on stale hosts should be migrated to the newly joined hosts. Hence the collaborative system has been reconfigured. The distributed object-oriented method in Java and RMI has high portability and flexibility in heterogeneous and reconfigurable environment. Such a framework is also suitable to implement Internet-based computation.

In the following text, Section 2 illustrates the collaborative system model and Section 3 describes the distributed object-oriented $N$-body method based on the collaborative system model. Section 4 reports the performance of the $\mathrm{N}$-body method on both homogeneous and heterogeneous clusters. Related work is covered in Section 5. The conclusions are summarized in Section 6.

\section{Collaborative System}

The $N$-body method is executed by a group of distributed objects on clustered hosts. A collaborative system is formed by the distributed objects at the beginning of the computation. Such a system can be reconfigured during the computation in response to the changes in the states of the hosts.

\subsection{System Establishment}

Fig. 2 shows the structure of a collaborative system. The compute coordinator is the first object created on one of the hosts to activate the computation. It finds the available hosts in cluster based on the information supplied by ClusterProbe - a Java-based tool for monitoring a large cluster, developed by the HKU System Research Group [8]. ClusterProbe is an open environment built on its multi-protocol communication interface that can receive various types of external accesses. It can scale up to work on large-sized cluster. It is a flexible monitoring tool which can adapt to the resource alternation in a cluster. ClusterProbe runs on a server. It provides the services for monitoring and management on all hosts in the cluster. One of its services is reporting the available hosts and their states, e.g. the workload. The compute coordinator selects a number of hosts to take part in the execution of an application based on the system information supplied by ClusterProbe. The hosts with low workload have priority to be chosen first. The compute coordinator starts computing objects on these hosts. The computing objects register themselves to RMI registry. After the registration, an object can get the references to all remote objects for interaction by looking up the registry. So a collaborative system is set up. It is ready to execute an application.

After the collaborative system has been established, the compute coordinator will divide the application into computing tasks and allocate the tasks to remote objects (compute engines), one task per compute engine, by the way of remote method invocation. The compute coordinator is also responsible to manage the collaborative system. It coordinates the computing procedure on all compute engines. The computing procedure is application-dependent. The communications between the compute engines are via method invocation interface of RMI. In the $N$-body method described in section 3 , the compute coordinator decomposes the physical domain and partitions $N$ bodies into subsets. It assigns the body subsets to remote compute engines. After the initiation of the computation, the compute coordinator also works as a compute engine to process one of the tasks. 


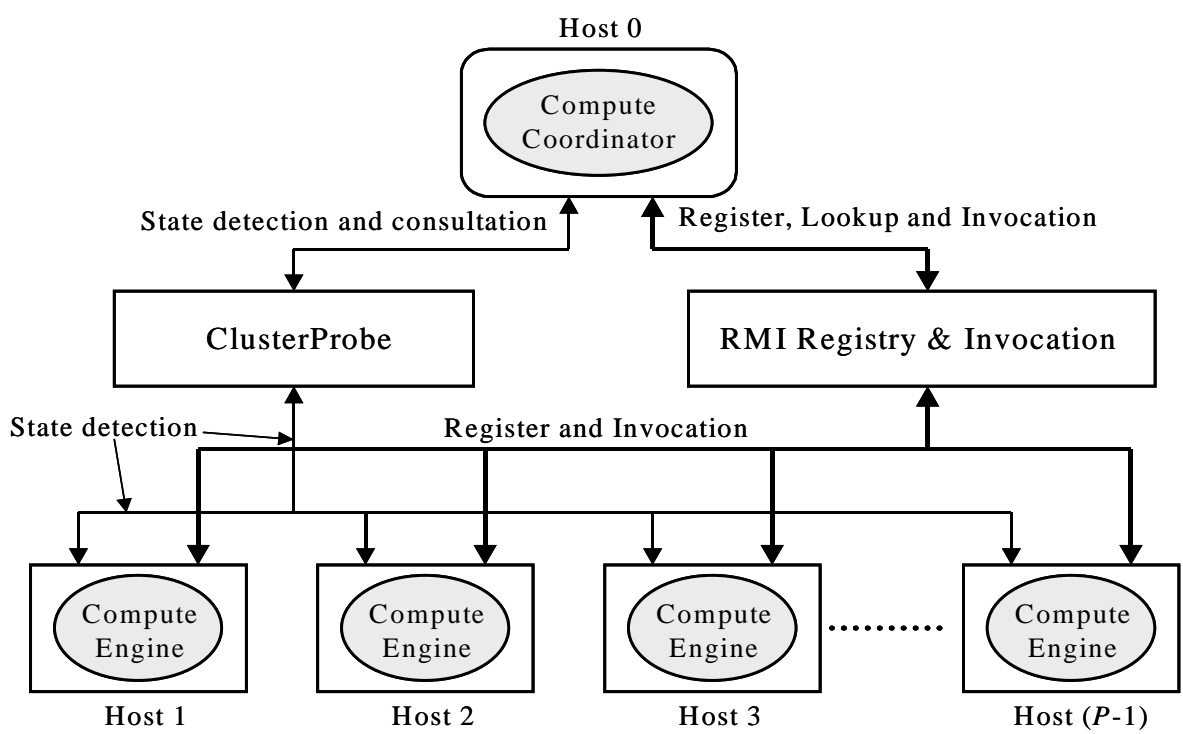

Fig. 2 The collaborative system built on $P$ hosts

\subsection{System Reconfiguration}

The configuration of a cluster can be dynamically altered. Some hosts may be removed from the cluster and new hosts may be added to the cluster. As the hosts in a cluster are shared by multiple users or by multiple jobs, the states of the hosts such as the workload may vary from time to time. Only the idle or lightly-loaded hosts are the proper candidates to join the collaborative system. ClusterProbe constantly detects the states of the hosts. The compute coordinator can reorganize the collaborative system according to the current status of the underlying cluster. It retrieves the latest status information from ClusterProbe and selects the lightly-loaded hosts to replace the overloaded ones in collaborative system. Computing objects are created on the newly-joined hosts. These objects become the compute engines by registering themselves to RMI registry. The compute engines on replaced hosts send their objects to the correspondent new compute engines by peer-to-peer communication. Afterwards the stale compute engines stop to work and the new compute engines take the place. Therefore the collaborative system has been reconfigured and the computation goes on. The system reconfiguration can improve the overall performance of the collaborative system.

In the $N$-body method, the particle simulation proceeds by iterating the simulation step. There is a proper opportunity to reconfigure the collaborative system at the end of each simulation step. At that moment, the compute coordinator checks the workload of the underlying hosts. If any host is overloaded, the compute coordinator will try to find a lightly-loaded host in the cluster for replacement. If the substitute host is available, the overloaded host will be replaced. The compute coordinator manages to create the compute engine on the new host. The new compute engine on the new host reads the body set on the replaced host. The new compute engine takes over the computing task from the overloaded host. The replaced compute engine ceases to work. The host substitution may happen on several overloaded hosts.

\section{DOO-based $N$-body Method}

The distributed object-oriented $\mathrm{N}$-body method originated from the Barnes-Hut application in SPLASH2 suite [14]. In our DOO method, a distributed tree structure is designed to reduce the communication overhead caused by global data sharing among the distributed objects. 


\subsection{Distributed Tree Structure}

The distributed tree structure is related to the domain partitioning. If there are totally $N$ bodies and $P$ hosts involved in the computation, the physical space should be partitioned into $P$ sub-domains and thus the $N$ bodies into $P$ subsets, one subtree for one sub-domain. The distributed data structure contains $P$ subtrees. The sub-domain partitioning is accomplished by decomposing the global BarnesHut tree that includes all bodies in the physical space. The partitioning should guarantee the spatial locality of bodies in sub-domain, and the number of bodies in each sub-domain should be approximately balanced. If the method is indicated to run on four hosts, the physical space in Fig. 1(a) will be partitioned into four sub-domains as Fig. 3(a) shows. Each compute engine will get a subset of bodies and build a local subtree for the sub-domain. So the distributed tree structure in Fig. 3(b) is composed of four subtrees.

The force computation of each body, however, requires the data from all other sub-domains. A compute engine needs to access not only its local subtree but also remote subtrees. There are two extreme approaches to share the data of other sub-domain. One is propagating the $N$ bodies to all compute engines and each compute engine builds a complete Barnes-Hut tree. So the computation on each engine can be locally fulfilled. It can be called complete tree approach. Another approach is sending the bodies to remote compute engines to access the subtrees there. The force contributions from those subtrees are computed on the remote compute engines and the results will be sent back to the home compute engine of the bodies. Obviously both of the two approaches will bring about prohibitive communication overhead.

In our $N$-body method a compromise is made to obtain partial sharing of the subtrees, i.e., broadcasting parts of subtrees to other compute engines. After the subtree has been constructed, every compute engine builds a part copy of the subtree called partial subtree as Fig. 3(b) shows. The partial subtree contains the top levels of the local subtree. The partial subtree will be broadcast to all other compute engines for their local use. Due to the partial duplication of the subtrees, most of the force computation can be completed on local compute engine. Only when a body requires the data in the lower levels of a remote subtree, it will be sent to the remote compute engine. In this case, the force contribution from that sub-domain is calculated on the remote compute engine by accessing the full subtree there. The result is sent back to the body's home compute engine. The partial subtree scheme can largely reduce the communication overhead in collaborative system. The benefit will be proved by the performance tests in Section 4.

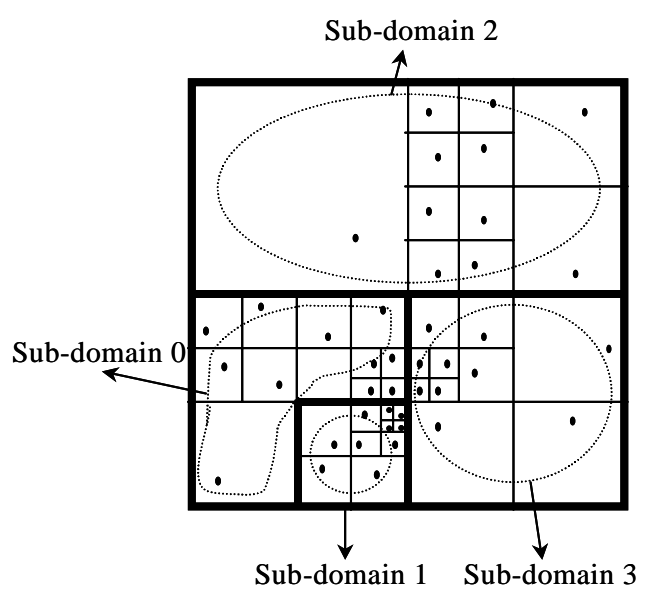

(a) Sub-domains after partitioning

(b) Distributed Barnes-Hut tree and partial subtrees 


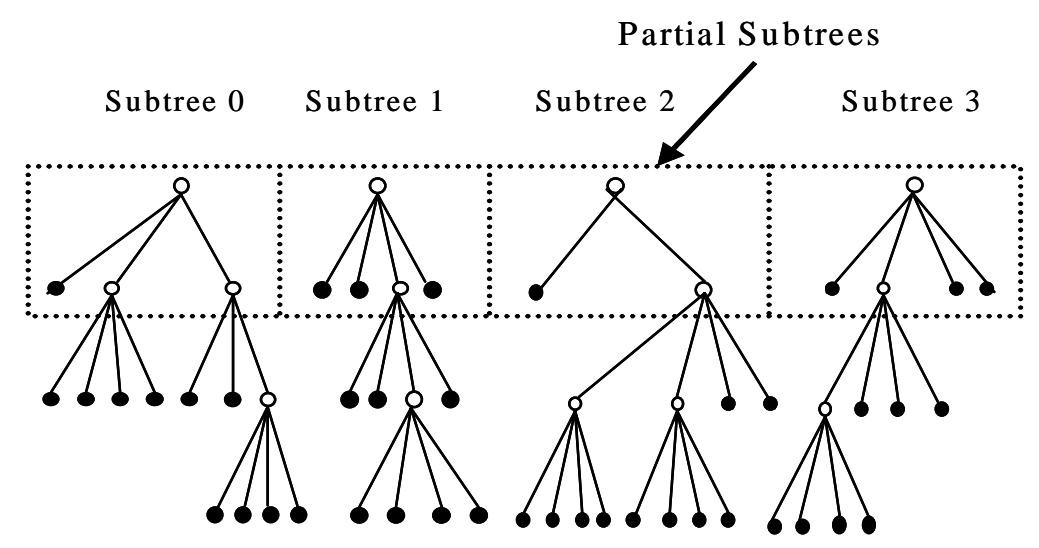

Fig. 3 A distributed Barnes-Hut tree for 2D space on four compute engines

\subsection{Computing Round}

The N-body method begins the particle simulation when the subsets of the bodies have been allocated to the remote compute engines. The simulation procedure advances by iterating the following computing round on all compute engines. A computing round represents one simulation step. The compute coordinator makes the synchronization of the computing round. A computing round consists of four substeps:

\section{Subtree construction and propagation}

Each compute engine builds a subtree and a partial subtree for the sub-domain assigned to it. The partial subtree is broadcast to other compute engines. So each compute engine has got all partial subtrees.

\section{Force calculation}

Each compute engine calculates the force exerting on every body in its sub-domain by traversing the local subtree and all partial subtrees. The bodies requiring to access remote subtrees are sent to the remote compute engines. In this case, the force contributions from the remote sub-domains are computed on remote compute engines by traversing the full subtrees there. The results are sent back to the bodies' home compute engines. Then the force contributions from all sub-domains are summed to get the total force influence on a body. Finally the states of the bodies are updated as the force effect, e.g. altering velocity and moving to new position.

\section{Body redistribution}

Each compute engine has been assigned a sub-domain at the beginning. So the boundary of each subdomain is known. At the end of substep 2, each body moved to its new position. Some bodies may exceed the scope of local sub-domain and enter adjacent sub-domains. It is needed to check the now position of every body to determine in which subdomain the new position locates. If it is beyond the local subdomain, the body should be transmitted to the destination compute engines where the new position drops in, so that the body locality in the subdomain can be maintained. As the bodies move ahead in small pace, there are usually only a few bodies transmitted in each computing round. So far a simulation step has finished.

\section{Load inspection and system reconfiguration (if necessary)}

Having finished a simulation step, the compute coordinator checks the hosts' workload by referring to the state information supplied by ClusterProbe. If the workload on a host exceeds the pre-defined threshold, the host is considered as overloaded. The coordinator will try to find another host in the cluster to replace the overloaded one. The substitute host should have the workload below the 
threshold and it has not joined the collaborative system yet. If the substitute is available, a computing engine will be created on it. The new compute engine registers itself to the collaborative system. The computing task on the overloaded host is migrated to the new host. In the $N$-body method, the computing task is represented by the bodies of a subdoamin. So all bodies set on the overloaded host is sent to the new host. Then the compute engine on the old host is terminated. The host replacement may take place on several overloaded hosts. The collaborative system is then reconfigured with the newly-joined compute engines and all remaining engines. The simulation procedure continues to start next computing round.

\section{Performance Test}

The $N$-body method is implemented in Java and RMI. It has been tested on two clusters. One is a homogeneous cluster, and the other is heterogeneous. The $N$-body application in the test simulates the three-dimensional motion of particles in Plummer distribution. Speedup can be achieved on both of the clusters. The platform-independent feature of Java programming supports the $N$-body application to be executable on the heterogeneous cluster.

\subsection{Speedup on Homogeneous Cluster}

The homogeneous cluster is a local cluster of PCs, including PentiumII $450 \mathrm{MHz}$ and Celeron $300 \mathrm{MHz}$ running Linux 2.0.36 or 2.2.1, connected by $100 \mathrm{Mbps}$ Fast Ethernet switch.

To manifest the lower communication overhead contributed by the distributed tree structure scheme, we implemented another straight-forward approach as a contrast. It is the complete tree approach: the compute coordinator starts a computing round by broadcasting all of the bodies to every compute engine, where a complete tree of $N$ bodies is built, like the Barnes-Hut tree in Fig. 1(b). In the complete tree scheme, each compute engine still computes the forces on a subset of bodies. But the force calculation can be totally finished within local compute engine. No remote tree access is required. Then the new states of $N$ bodies from remote compute engines are broadcast to all compute engines again for next computing round.

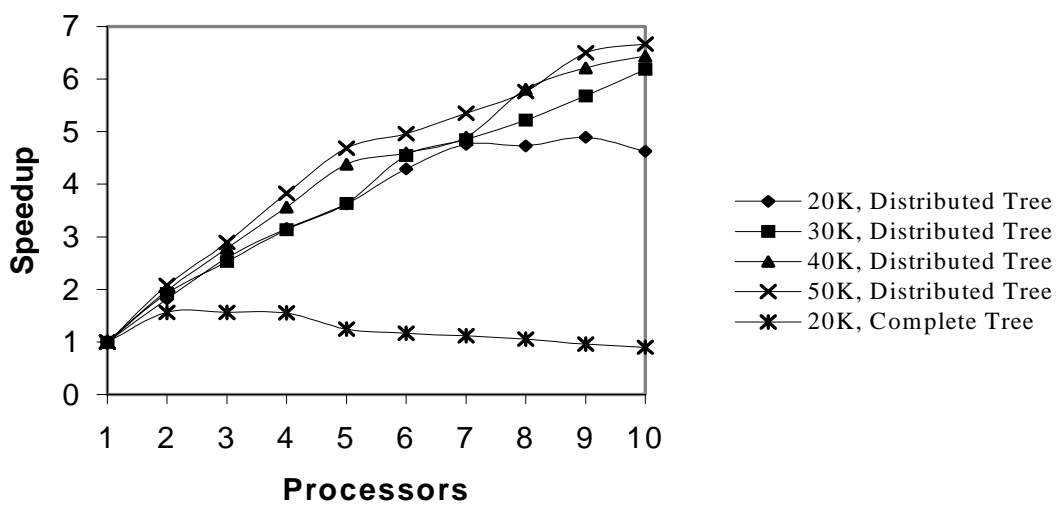

Fig. 4 The speedup of two $N$-body methods (distributed tree and complete tree approach) on the homogeneous cluster of PCs, under different problem size $N$ 


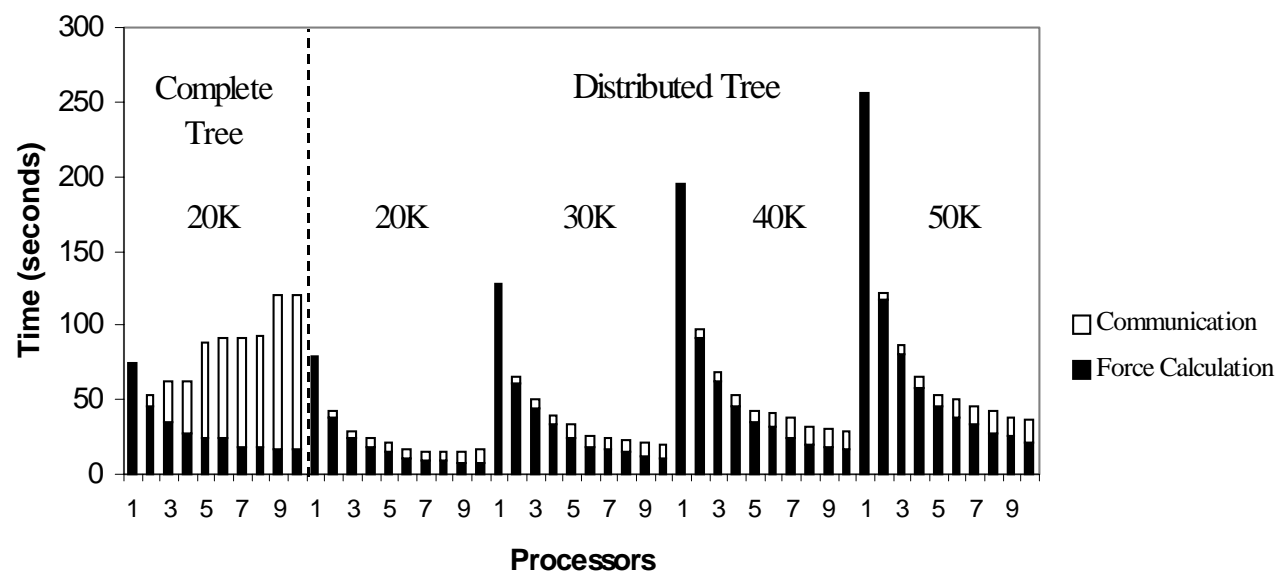

Fig. 5 The execution time breakdowns of $N$-body methods

As the data communication overhead is much higher in the complete tree approach than that in distributed tree scheme, the communication overhead in complete tree approach is much higher.

Fig. 4 displays the speedup of the $N$-body method with distributed tree structure and the complete tree approach. For the distributed tree method, speedup can be obtained on one to ten processors. Larger speedup can be achieved on larger problem size. On the other hand, broadcasting $N$ bodies inhibits the speedup of the complete tree approach. The speedup occurs merely below three hosts. The execution slows down when more processors are used.

The time breakdowns in Fig. 5 show the communication efficiency of the distributed tree structure. In distributed tree method, the communication occupies a small proportion of the execution time. But in complete tree approach, most of the execution time is spent in communication on three hosts or above. The test results reveal that the distributed tree is an appropriate data structure for distributed $N$-body method.

\subsection{Platform-independence on Heterogeneous Cluster}

The $N$-body method on distributed tree structure has also been tested on a wider-range heterogeneous cluster. The cluster consisted of six hosts:

- Two Pentium II 450MHz PCs, running Linux 2.0.36;

- Two Sun UltraSPARC-1 workstations, running Solaris 2.6;

- Two processors in an SGI PowerChallenge SMP, running IRIX 6.2.

This is a heterogeneous system with three types of processors. These hosts located at different sites. Two PentiumII PCs lay in the local cluster as in the former test. Two Sun UltraSPARC-1workstations belonged to a remote cluster of Sun workstations, and the SGI PowerChallenge was a remote server. The hosts were linked together across HKU campus network. The $N$-body application in Java and RMI could uniformly run on these platforms without any modification. The application ran on one to six processors in the order of two Pentium II PCs, two Sun UltraSPARC-1 workstations, and two processors in SGI PowerChallenge. Fig. 6 (a) shows the speedup of the distributed tree method on the heterogeneous cluster. The sequential time was measured on a Pentium II PC. Speedup can be observed on the heterogeneous cluster too.

The execution time breakdown on the heterogeneous cluster shown in Fig. 6(b) resembles that on the homogeneous cluster. The communication latency was a bit longer due to the heavy communication traffic in campus network. However, the communication time is still shorter than the computation time. The result has further verified the communication efficiency of the distributed tree structure. The 
collaborative system model is an appropriate framework for distributed computing in networked environment.

It has been observed that the execution time of Java application is not satisfactory because of the inefficiency of Java interpreter. Nevertheless Java possesses the attractive properties as strong platform-independent portability and flexibility that are demanded in network computing. In addition, the performance of Java application is getting improved along with the upgrading Java system.

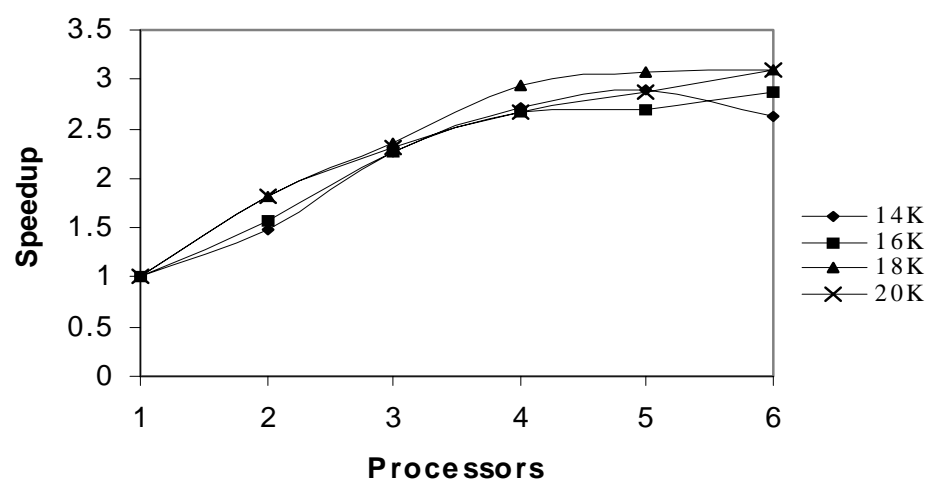

(a) Speedup of the $N$-body method in distributed tree scheme with different problem size $N$

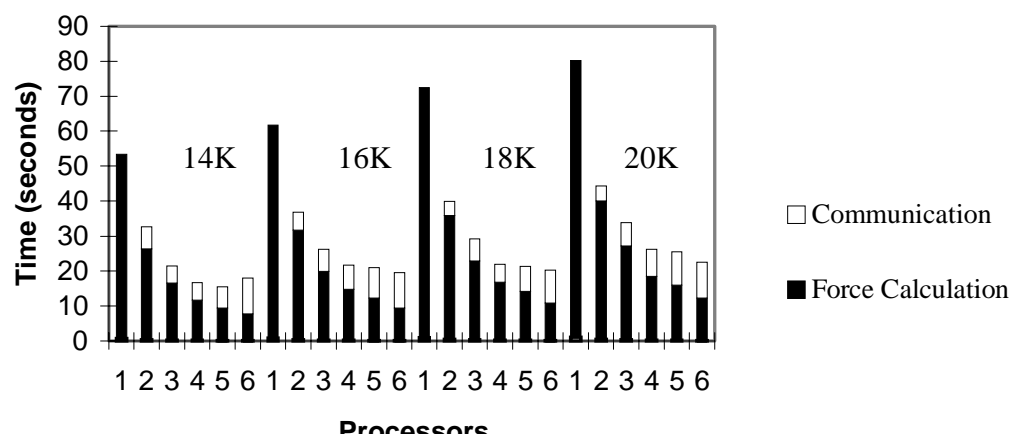

(b) Breakdown of execution time

Fig. 6 The speedup and the execution time breakdown of the $N$-body method (distributed tree structure) on the heterogeneous cluster

\section{Related Work}

There are other instances of parallel $N$-body methods based on Barnes-Hut method. This section introduces three examples.

Grama presented a parallel implementation of the Barnes-Hut method on message passing computer [4]. In his method, a 2D physical domain was partitioned into subdomains. The particles in one of the subdomains were assigned to one processor. A local tree was constructed per processor and then all local trees were merged to form a global tree. All nodes above a certain cut-off depth in the global tree 
were broadcast to all processors. Grama's method for 2D space was run on a 256-processor nCUBE2 parallel computer. In our DOO method, however, there is no global tree to be built but the top levels of the subtrees, i.e. partial subtrees, are broadcast. Our scheme can save the cost of building a global tree and increase the ratio of force computation on local compute engine. Our method is more appropriate in distributed environment.

The other two methods are the applications built on a general-purpose data structure layer and implemented in $\mathrm{C}++$.

The object-oriented support for adaptive methods in [2] provided a global data structure PTREE that was implemented as a collection of local data structures on distributed-memory machine. The data structure was distributed to multiple processors where computations were carried out and the partial results were merged. The global data structure could support different applications. A gravitational $\mathrm{N}$ body simulation was implemented on the global data structure. The application was tested on a 64-node iPSC/860 machine.

Liu [9] described an implementation of parallel $C++N$-body framework that could support various scientific simulations which involved tree structures. The framework consisted of three layers: (1) generic tree layer supported simple tree construction and manipulation methods, and system programmers could build special libraries using classes in the layer. (2) Barnes-Hut tree layer supported tree operations required in most of the $N$-body tree algorithms. (3) application layer implemented a gravitational $\mathrm{N}$-body application upon the $\mathrm{BH}$-tree layer. The communication library was implemented in MPI. The application was executed on a cluster of four Ultra Sparc workstations connected by a fast Ethernet network.

On the contrary, our method is based on a distributed tree structure dedicated to DOO $N$-body method. The method should be more efficient on distributed computing. Furthermore our $N$-body application is implemented in Java and RMI. It is executable on heterogeneous system and adaptive to system reconfiguration. So it is a more flexible method on various platforms.

\section{Conclusions}

We have discussed a distributed object-oriented (DOO) method for solving $N$-body problems on cluster. Distributed objects on different hosts form a collaborative system to execute the application. The method is implemented in Java and RMI interface to support distributed computing, especially on heterogeneous platforms. By RMI registry mechanism for distributed objects, the collaborative system can be dynamically reconfigured during the computation to fully utilize the computing resources in a cluster. The collaborative system model, with the architectural-neutral features of Java and RMI, has high flexibility, portability and adaptability on different platforms. The collaborative system is also feasible for other applications. It is a promising framework for distributed computing in heterogeneous environment. The framework can also be expanded to wide-area environment.

$N$-body method deals with a great number of particles in physical space. There exists high data dependency among all bodies. The heavy communication is the bottleneck for the performance. We propose a distributed tree structure to reduce communication overhead. The $N$-body method has been tested on a local homogeneous cluster and a wider-range heterogeneous cluster. The results of performance tests show that the DOO method is appropriate in distributed and heterogeneous system.

\section{References}

[1] J. Barnes and P. Hut, A Hierarchical $O(N \log N)$ Force-Calculation Algorithm, Nature 324(4) (1986) 446-449.

[2] S. Bhatt, M. Chen, and et al., Object-Oriented Support for Adaptive Methods on Parallel Machines, Scientific Computing 2 (1993) 179-192.

[3] J. Farley, Java Distributed Computing, (O’Reilly \& Associates Inc, USA, 1998)

[4] A.Y. Grama, V. Kumar and A. Sameh, n-body Simulation Using Message Passing Parallel Computers, Proceedings of the $7^{\text {th }}$ SIAM Conference on Parallel Processing for Scientific Computing (1995) 355-360. 
[5] L. Greengard and V. Rokhlin, A Fast Algorithm for Particle Simulations, Journal of Computational Physics 73 (1987) 325-348.

[6] L. Hernquist, Hierarchical N-body Methods, Computer Physics Communications 48 (1988) 107-115.

[7] Y.C. Hu, S.L. Johnsson and S.H. Teng, A Data-Parallel Adaptive $N$-body Method, Proceedings of $8^{\text {th }}$ SIAM Conference on Parallel Processing for Scientific Computing (1997)

[8] Z. Liang, Y. Sun, and C.L. Wang, ClusterProbe: An Open, Flexible and Scalable Cluster Monitoring Tool, First International Workshop on Cluster Computing, Australia, August, 1999.

[9] P. Liu and J.J. Wu, A Framework for Parallel Tree-Based Scientific Simulations, Proceedings of $26^{\text {th }}$ International Conference on Parallel Processing (1997)

[10] J. Salmon and M.S. Warren, Parallel, Out-of-core methods for $N$-body Simulation, Proceedings of the $8^{\text {th }}$ SIAM Conference on Parallel Processing for Scientific Computing (1997).

[11] J.P. Singh, J.L. Hennessy and A. Gupta, Implications of Hierarhical N-Body Methods for Multiprocessor Architectures, ACM Transactions on Computer Systems 13(2) (1995) 141-202.

[12] J.P. Singh, C. Holt and et al., Load Balancing and Data Locality in Adaptive Hierarchical $N$-body Methods: Barnes-Hut, Fast Multipole, and Radiosity, Journal of Parallel and Distributed Computing 27(2) (1995) 118141.

[13] Y. Sun, Z. Liang and C.L. Wang, A Distributed Object-Oriented Method for Particle Simulations on Clusters, Proc. HPCN'99, LNCS, Vol.1593 (Spriner, Berlin, 1999) 251-259.

[14] S.C. Woo, M. Ohara and et al., The SPLASH-2 Programs: Characterization and Methodological Considerations, Proceedings of $22^{\text {nd }}$ Annual International Symposium on Computer Architecture (1995). 